\title{
PERFORMANCE OPTIMIZATION OF A MANEUVERING RE-ENTRY VEHICLE USING A LEGENDRE PSEUDOSPECTRAL METHOD
}

\author{
Anil V. Rao* \\ Kimberley A. Clarke ${ }^{\dagger}$ \\ The Charles Stark Draper Laboratory, Inc. \\ Cambridge, MA 02139-3563
}

\begin{abstract}
The problem of performance optimization of a maneuvering re-entry vehicle is studied. The objective is to determine an optimal steering law for a high lift-to-drag ratio vehicle. An optimization criteria is derived that attempts to maximize the control margin of the vehicle in order to account for unmodeled perturbations during actual flight. The optimization problem is formulated as an optimal control problem. The optimal control problem is transcribed to a nonlinear programming via a Legendre pseudospectral method and is solved using a sparse nonlinear optimization algorithm. The key features of the optimal trajectory are described and the quality of the trajectory obtained from the Legendre pseudospectral method is discussed. Finally, a motivation is given for using the pseudospectral method as a guidance law.
\end{abstract}

\section{INTRODUCTION}

Developing guidance algorithms for next-generation atmospheric and space flight vehicles is a great challenge because these new vehicles will have significantly higher performance requirements over current generation vehicles. In order to achieve the required performance, the on-board guidance algorithm must be capable of exploiting a large portion of the vehicle capability. Furthermore, the guidance law must be robust to environmental perturbations. At first glance it may appear most cost-effective to develop new guidance laws that are based on those currently in use. However, current guidance algorithms are not even capable of exploiting the full capability of those vehicles on which they are used and hence it is difficult to conceive of extending the current guid- ance technology to next-generation vehicles. Consequently, the next generation of aerospace vehicles will require more advance guidance methodologies.

A particular class of next-generation atmospheric flight vehicles that will require advanced guidance is the class of unmanned maneuvering re-entry vehicles ${ }^{1}$ where the objective is to steer the vehicle from atmospheric entry to a terminal state whose position must be achieved with great accuracy, whose impact speed must be high and must lie within a narrow range, whose flight path angle must be nearly orthogonal to the plane of the target, and whose angle of attack must be nearly zero. ${ }^{3}$ In addition, because vehicles in this class have one-sided angle of attack control (i.e. the angle of attack must remain positive throughout flight), environmental perturbations that occur during actual flight further increase the demands on the guidance law to provide a control that is capable of steering the vehicle to the target. Because of the

* Senior Member of the Technical Staff, Guidance and Navigation Division, 555 Technology Square, Mail Stop 70, Cambridge, MA 02139-3563. E-mail: arao@draper.com. Corresponding Author

${ }^{\dagger}$ Draper Laboratory Fellow, Guidance and Navigation Division. Graduate Student, Department of Aeronautics and Astronautics, Massachusetts Institute of Technology. E-mail: kclarke@draper.com

Copyright 2002 by The Charles Stark Draper Laboratory, Inc. Published by the American Institute of Aeronautics \& Astronautics, Inc., with permission. 
aforementioned limitations of current guidance algorithms, it is desirable to developed advanced guidance approaches for use on board a high performance unmanned maneuvering re-entry vehicle.

In general, guidance law development is typically split into two phases. The first phase is the design of a so called reference trajectory and reference control. The reference trajectory and reference control are often (but not always) computed by solving a mission-specific optimal control problem. After obtaining a reference trajectory, one of two approaches are commonly used to design a guidance law. The first approach is called trajectory tracking where the objective of the guidance law is to follow the reference trajectory as closely as possible. The second approach is called terminal guidance where the control is updated periodically (where the period is called a guidance cycle) and the control is chosen at each guidance cycle so as to steer the vehicle towards the target. Moreover, in terminal guidance the control is generally obtained in a "near-optimal" manner, i.e. at each guidance cycle the control is chosen to approximate the instantanous solution of the optimal control problem at that instant of time. Trajectory tracking is used when it is not only important for the vehicle to reach the target, but it is also important that the vehicle fly along or near a particular path in the state space. Terminal guidance is used primarily when the particular path is not important (except that the trajectory remain feasible), but that the vehicle attains the desired terminal state. In this paper we are interested in terminal guidance.

While ideally a terminal guidance law would be capable of re-solving the optimal control problem at each guidance cycle, such an idealization has not been realizable due to limitations in computational resources (i.e. slow processor speed and limited memory). However, computational resources have increased significantly over the past several years, and these resources increasing at a rapid rate each year. This increase in computational power offers an opportunity to develop more sophisticated guidance guidance algorithms. In the context of terminal guidance, the increase in computational power makes solving an optimal control problem in real time within the realm of possibility. Therefore, it is useful to conceive of methods for solving optimal control problems that have the potential of being used in real time.

In general, optimal control problems for atmospheric and space flight vehicles are nonlinear and do not have analytic solutions. Consequently, it is necessary to solve these optimal control problems numerically. Numerical methods for optimal control fall into two distinct categories: indirect methods and direct methods. In an indirect method, extremal trajectories are found by solving a Hamiltonian boundaryvalue problem (HBVP) derived from the first-order necessary conditions for optimality. A benefit of finding a solution to the HBVP is that an accurate costate is obtained from which an accurate control can be computed. However, many HBVPs of interest in atmospheric flight and space flight suffer from extreme sensitivity to unknown boundary conditions. Consequently, it is often difficult to find solutions to HBVPs which in turn makes it difficult to implement an indirect method in real time.

In a direct method the optimal control problem is transcribed to a nonlinear programming (NLP) problem by discretizing the differential equations at specific time points called nodes. The NLP is then solved using an appropriate numerical optimization algorithm (see Ref. 4 and the references contained therein). Direct methods have proven to be significantly more capable than indirect methods at solving a wide range of complex optimal control problems, even when a poor initial guess is provided for the numerical optimization algorithm. An excellent survey of various numerical methods for solving trajectory optimization problems can be found in Ref. 9. Well-known software packages employing direct methods include Optimal Trajectories by Implicit Simulation (OTIS) ${ }^{10}$ and Sparse Optimal Control Software (SOCS). ${ }^{5}$ The reason that direct methods are superior to indirect methods in their solution capability is that finding a solution to the NLP is easier than finding a solution to the corresponding HBVP. However, as a consequence of the choice of the discretization of the differential equations, the co-state obtained from many direct methods is significantly less accurate than the co-state obtained via an indirect method. Consequently, the control obtained from a direct method is generally less accurate than the control obtained using an indirect method. Because of this inaccuracy, it is difficult to employ a direct method in real time.

It can be seen that a method for the real-time solution of optimal control problems useful in the context of guidance is one that possesses desirable features of both indirect and direct methods, i.e. a desirable method is one that is capable of solving a wide range of problems and simultaneously provides accurate co-state information. A newly developed class of methods for solving optimal control problems that has positive attributes of both indirect and direct methods is the class of so-called pseudospectral methods. ${ }^{6-8}$ In a pseudospectral method, the state and control are discretized at specified time points (called nodes) using a basis of global orthogonal poly- 
nomials. Using this choice of transcription and the proper choice of the nodes, an efficient discretization is obtained from which an accurate approximation is obtained to the optimal trajectory of the continuoustime optimal control problem. Simultaneously, an accurate co-state can be obtained from which an accurate approximation to the optimal control can be computed.

A particular pseudospectral method than has shown promise for use in real time is the so-called Legendre Pseudospectral Method developed by Fahroo and Ross. ${ }^{6}$ In this method the state and control are parameterized as a linear combination of the Lagrange polynomials and the nodes are the LegendreGauss-Lobatto points. A key feature of the Legendre pseudospectral method is that an accurate costate of the continuous-time optimal control problem is obtained via a known scaling of the Karush-KuhnTucker (KKT) Lagrange multipliers of the NLP. The fact that a high quality co-state can be obtained from the Legendre pseudospectral method makes it a viable candidate for use in guidance.

In this paper, the Legendre pseudospectral method of Ref. 6 is applied to optimal reference trajectory and reference control design for an unpowered maneuvering re-entry vehicle. A performance index for the optimal control problem is constructed that attempts to maximize control margin. It is shown that the Legendre pseudospectral method performs extremely well on this problem, providing a reference trajectory and control that are extremely close to the extremal trajectory and control predicted by the firstorder necessary conditions for optimality. Finally, the potential for using the Legendre pseudospectral method as a guidance law is discussed.

\section{REFERENCE TRAJECTORY DESIGN}

\section{Equations of Motion \& Physical Model}

The maneuvering re-entry vehicle is modeled as a point mass flying over a spherical rotating Earth. The motion of the vehicle is modeled using spherical co- ordinates and is taken verbatim from Ref. 2 .

$$
\begin{aligned}
& \dot{r}=v \sin \gamma, \quad \dot{\theta}=\frac{v \cos \gamma \cos \psi}{r \cos \phi}, \quad \dot{\phi}=\frac{v \cos \gamma \sin \psi}{r} \\
& \dot{v}=-D-g \sin \gamma+\omega^{2} r \cos \phi(\sin \gamma \cos \phi-\cos \gamma \sin \psi \sin \phi) \\
& \dot{\gamma}=\frac{L \cos \sigma}{v}-\left(\frac{g}{v}-\frac{v}{r}\right) \cos \gamma+2 \omega \cos \psi \cos \phi \\
& +\frac{\omega^{2} r}{v} \cos \phi(\cos \gamma \cos \phi+\sin \gamma \sin \psi \sin \phi) \\
& \dot{\psi}=\frac{L \sin \sigma}{v \cos \gamma}-\frac{v}{r} \cos \gamma \cos \psi \tan \phi \\
& +2 \omega(\tan \gamma \sin \psi \cos \phi-\sin \phi) \\
& -\frac{\omega^{2} r}{v \cos \gamma} \cos \psi \sin \phi \cos \phi
\end{aligned}
$$

where $r$ is the geocentric radius, $\theta$ is the Earth relative longitude measured East from the Prime Meridian, $\phi$ is the geocentric latitude measured positively North from the equatorial plane, $v$ is the Earth relative speed, $\gamma$ is the Earth relative flight path angle, $\psi$ is the heading angle, and $\sigma$ is the bank angle. The bank angle $\sigma$ defines the angle between the lift vector and the $(\boldsymbol{r}, \boldsymbol{v})$ plane where $\boldsymbol{r}$ is the position and $\boldsymbol{v}$ is the Earth relative velocity. Finally, the rotation rate of the Earth is denoted by $\omega$ and $g=\mu / r^{2}$ is the magnitude of the gravitational acceleration where $\mu$ is the gravitational parameter for the Earth. The lift acceleration, $L$, and drag acceleration, $D$, are given as

$$
\begin{aligned}
& L=q S C_{L} / m \\
& D=q S C_{D} / m
\end{aligned}
$$

where $q=\rho v^{2} / 2$ is the dynamic pressure, $C_{L}$ is the coefficient of lift, $C_{D}$ is the coefficient of drag, $S$ is the reference area of the vehicle, $m$ is the vehicle mass, and $\rho$ is the atmospheric density. The density is given as

$$
\rho=\rho_{0} \exp (-\beta h)
$$

where $\rho_{0}$ is the density at sea level, $h=r-R_{e}$ is the altitude, $R_{e}$ is the radius of the Earth, and $\beta$ is the inverse of the density scale-height. The aerodynamic model used in this analysis is given as ${ }^{1,2}$

$$
\begin{array}{rrr}
C_{D} & = & C_{D 0}+K C_{L}^{2} \\
C_{L} & = & C_{L, \alpha} \alpha
\end{array}
$$

where $C_{D 0}$ is the zero-lift drag coefficient, $K$ is the drag polar parameter, $\alpha$ is the angle of attack, and $C_{L, \alpha}$ is a constant. The physical constants are taken from Ref. 11 while the following values are used for the aerodynamic parameters:

$$
\begin{array}{lrr}
C_{D 0} & = & 0.043 \\
K & = & 1 \\
C_{L, \alpha} & = & 1
\end{array}
$$


The aerodynamic parameters in Eq. (5) represent a high lift-to-drag ratio vehicle.

Since it is not possible in practice to apply an infinite rate control, the controls $\alpha$ and $\sigma$ are ratelimited as follows by augmenting the following two differential equations to the dynamics of Eq. (1):

$$
\begin{aligned}
& \dot{\alpha}=u_{\alpha} \\
& \dot{\sigma}=u_{\sigma}
\end{aligned}
$$

Furthermore, bounds are placed on $\left|u_{\alpha}\right|$ and $\left|u_{\sigma}\right|$. The specific bounds are given later in the discussion.

\section{Path Constraints}

During flight, inequality constraints are imposed on dynamic pressure, sensed acceleration, angle of attack, and the control rates $u_{\alpha}$ and $u_{\sigma}$. The sensed acceleration is defined as

$$
a=\sqrt{D^{2}+L^{2}}
$$

The dynamic pressure is bounded below by a predefined value $q_{\min }$ while the sensed acceleration is bounded above by a pre-defined value $a_{\max }$. Furthermore, the angle of attack is constrained to lie between zero and a pre-defined value $\alpha_{\max }$. Finally, absolute values of the control rates, $\left|u_{\alpha}\right|$ and $\left|u_{\sigma}\right|$ are bounded by positive constants $u_{\alpha, \max }>0$ and $u_{\sigma, \max }>0$, respectively. Consequently, the following inequality constraints are imposed during flight:

$$
\begin{array}{llr}
q & \geq & q_{\min } \\
a & \leq & a_{\max } \\
\alpha & \geq & 0 \\
\alpha & \leq & \alpha_{\max } \\
\left|u_{\alpha}\right| & \leq & u_{\alpha, \max } \\
\left|u_{\sigma}\right| & \leq & u_{\sigma, \max }
\end{array}
$$

\section{Boundary Conditions}

The boundary conditions for the maneuvering reentry vehicle problem are as follows: (1) The initial position and velocity are completely specified, (2) The terminal position and velocity are completely specified, and (3) the terminal angle of attack must be zero. The terminal boundary condition on the angle of attack is called the obliquity condition. In terms of the components of the state, the initial conditions are given as

$$
\begin{aligned}
r\left(t_{0}\right) & =r_{0} \\
\theta\left(t_{0}\right) & =\theta_{0} \\
\phi\left(t_{0}\right) & =\phi_{0} \\
v\left(t_{0}\right) & =v_{0} \\
\gamma\left(t_{0}\right) & =\gamma_{0} \\
\psi\left(t_{0}\right) & =\psi_{0}
\end{aligned}
$$

where $t_{0}$ denotes the initial time. Similarly, the terminal conditions are given as

$$
\begin{aligned}
r\left(t_{f}\right) & =r_{f} \\
\theta\left(t_{f}\right) & =\theta_{f} \\
\phi\left(t_{f}\right) & =\phi_{f} \\
v\left(t_{f}\right) & =v_{f} \\
\gamma\left(t_{f}\right) & =\gamma_{f} \\
\psi\left(t_{f}\right) & =\psi_{f} \\
\alpha\left(t_{f}\right) & =0
\end{aligned}
$$

where $t_{f}$ denotes the terminal time.

\section{Objective Functional}

In order to allow the vehicle to correct for unpredictable flight conditions, an objective functional is chosen that attempts to maximize the amount of control margin during flight. The control margin is defined qualitatively as the distance that the control lies from its boundaries. Therefore, an objective functional is constructed that attempts to keep $\alpha$ near the middle of its capability. Thus, one term in the cost function is a penalty on on deviations in $\alpha$ from $\bar{\alpha}$ (where $\bar{\alpha}=\sqrt{C_{D 0} / K}$ corresponds to the angle of attack at the maximum value of $L / D$ ), and penalties on large control rates $u_{\alpha}$ and $u_{\sigma}$. Qualitatively, keeping the angle of attack near $\bar{\alpha}$ and keeping $\alpha$ away from $\alpha_{\max }$ allows the vehicle to either increase or decrease in the case of off-nominal perturbations during an actual flight. Keeping $u_{\alpha}$ and $u_{\sigma}$ small helps reduce the amount of actuator propellant required on board, thus reducing the mass of the vehicle. Consequently, the performance index chosen for this analysis is

$$
\begin{gathered}
\mathcal{J}=\int_{0}^{t_{f}}\left[k_{1}\left(\frac{\alpha-\bar{\alpha}}{\alpha_{\max }}\right)^{2}+k_{2}\left(\frac{u_{\alpha}}{u_{\alpha, \max }}\right)^{2}\right. \\
\left.+k_{3}\left(\frac{u_{\sigma}}{u_{\sigma, \max }}\right)^{2}\right] d t
\end{gathered}
$$

where $k_{1}, k_{2}$, and $k_{3}$ are design parameters. It is noted that the terms in the integrand of the cost functional of Eq. (11) are scaled by their respective maximum values (e.g. the first term is scaled by $\alpha_{\max }$ ) in order to more easily interpret the relative contribution of each term to the overall cost. A detailed study of this issue is beyond the scope of this paper.

\section{Optimal Control Problem}

The reference trajectory for the maneuvering re-entry vehicle under consideration in this paper is computed by solving the following optimal control problem: Find the control $\left(u_{\alpha}(t), u_{\sigma}(t)\right)$ on the time interval 
$t \in\left[0, t_{f}\right]$ (where $t_{f}$ is free) that minimize the objective functional of Eq. (11) subject to the differential equations of Eqs. (1) and (6), the path constraints of Eq. (8), and the boundary conditions described above.

\section{LEGENDRE PSEUDOSPECTRAL METHOD}

The aforementioned optimal control problem is solved using the Legendre pseudospectral method of Fahroo and Ross of Ref. 6. It is beyond the scope of this paper to provide a detailed description of the Legendre pseudospectral method. A brief summary of the method is provided here in order to maintain continuity. Please see Ref. 6 for further details of the method.

In the Legendre pseudospectral method of Ref. 6, the the continuous-time optimal control problem is transcribed to a nonlinear programming problem (NLP). For a given choice of nodes (where $N+1$ is the number of nodes), the NLP that results from the discretization is as follows: ${ }^{6}$ Minimize

$$
\mathcal{J}^{N}=\mathcal{M}\left(\boldsymbol{a}_{N}, \tau_{f}\right)+\frac{\tau_{f}-\tau_{0}}{2} \sum_{k=0}^{N} \mathcal{L}\left(\boldsymbol{a}_{k}, \boldsymbol{b}_{k}\right) w_{k}
$$

subject to the equality constraints

$$
\begin{gathered}
\frac{\tau_{f}-\tau_{0}}{2} \boldsymbol{f}\left(\boldsymbol{a}_{k}, \boldsymbol{b}_{k}\right)-\sum_{k=0}^{N} D_{k l} \boldsymbol{a}_{l}=\mathbf{0} \\
k=0,1, \ldots, N \\
\psi_{0}\left(\boldsymbol{a}_{0}, \tau_{0}\right) \equiv \mathbf{0} \\
\psi_{f}\left(\boldsymbol{a}_{N}, \tau_{f}\right) \equiv \mathbf{0}
\end{gathered}
$$

and the inequality constraints

$$
\boldsymbol{g}\left(\boldsymbol{a}_{k}, \boldsymbol{b}_{k}\right) \leq 0 \quad, \quad k=0,1, \ldots, N
$$

It is noted that in the method of Ref. 6, the nodes are the Legendre-Gauss-Lobatto (LGL) points. Please see Ref. 6 for further details.

\section{Co-State Estimation}

It has been shown in Ref. 6 that solving a continuoustime optimal control problem via the Legendre pseudospectral method leads to the following estimates for the co-states:

$$
\boldsymbol{\lambda}\left(t_{k}\right)=\widetilde{\boldsymbol{\lambda}}_{k} / w_{k}
$$

where $\boldsymbol{\lambda}\left(t_{k}\right)$ is the estimate the co-state for the continuous-time optimal at the $k^{\text {th }}$ LGL point, $\widetilde{\boldsymbol{\lambda}}_{k}$ is the corresponding KKT multiplier associated with the solution of the NLP of Eqs. (12)-(14), and $w_{k}$ is the LGL weight corresponding the $k^{\text {th }}$ LGL point. Eq. (15) will be used to demonstrate the accuracy of the solution of the continuous-time optimal control problem when solved via the Legendre pseudospectral method.

\section{APPLICATION OF PSEUDOSPECTRAL METHOD}

In order to obtain a well-scaled NLP, the following canonical units were chosen for numerical computation:
Units of Length: Earth Radii
Units of Time: Period of a Spacecraft
in Circular Orbit at
One Earth Radii
Units of Speed: Speed of a Spacecraft in
Circular Orbit at
One Earth Radii
Units of Density: Air Density at Sea Level

The results were then scaled to dimensionalized quantities for analysis. It is noted that the use of canonical units does not alter the mathematical form of the differential equations given in Eq. (1) and (6), only the numerical values for the physical constants are changed.

The specific boundary conditions used to obtain the results presented in this paper are based on values given in Ref. 3 and are given as follows:

$$
\begin{aligned}
t_{0} & =0 \mathrm{~s} \\
t_{f} & =\mathrm{FREE} \\
r\left(t_{0}\right) & =6378182 \mathrm{~m}(=37 \mathrm{~km} \text { altitude }) \\
\theta\left(t_{0}\right) & =0 \mathrm{deg} \\
\phi\left(t_{0}\right) & =0 \mathrm{deg} \\
v\left(t_{0}\right) & =7137.9 \mathrm{~m} / \mathrm{s} \\
\gamma\left(t_{0}\right) & =0 \mathrm{deg} \\
\psi\left(t_{0}\right) & =0 \mathrm{deg} \\
r\left(t_{f}\right) & =6378145 \mathrm{~m}(=0 \mathrm{~km} \text { altitude }) \\
\theta\left(t_{f}\right) & =25.15 \mathrm{deg}(=2800 \mathrm{~km} \text { downrange }) \\
\phi\left(t_{f}\right) & =0 \mathrm{deg} \\
v\left(t_{f}\right) & =1219 \mathrm{~m} / \mathrm{s} \\
\gamma\left(t_{f}\right) & =-89 \mathrm{deg} \\
\psi\left(t_{f}\right) & =\mathrm{FREE} \\
\alpha\left(t_{f}\right) & =0 \mathrm{deg}
\end{aligned}
$$


The boundary conditions of Eq. (16) correspond to a target that is approximately $2800 \mathrm{~km}$ downrange from the initial state (see Appendix for the definition of downrange). Furthermore, the target lies in the initial Earth relative trajectory plane. It is noted that in the current application the terminal velocity of the vehicle should be orthogonal to the plane that is tangent to the surface of the Earth at the target location. Strictly speaking, this orthogonality conditions requires that the terminal flight path angle be -90 deg. However, the system of differential equations of Eq. (1) chosen for this study has a singularity at $\gamma=-90 \mathrm{deg}$. Therefore, instead of setting the terminal flight path angle to $-90 \mathrm{deg}$, the value $\gamma_{f}=-89 \mathrm{deg}$ was used. Finally, because $\gamma_{f}=-89 \mathrm{deg}$ corresponds to a near-vertical orientation of the vehicle at Earth impact, it was not necessary to constrain the terminal heading angle (since the heading angle is undefined when the vehicle is flying vertically downward).

The constraints imposed on the control rates $u_{\alpha}$ and $u_{\sigma}$ are

$$
\begin{aligned}
& \left|u_{\alpha}\right| \leq 10 \mathrm{deg} / \mathrm{s} \\
& \left|u_{\sigma}\right| \leq 30 \mathrm{deg} / \mathrm{s}
\end{aligned}
$$

Furthermore, the path constraints are bounded as

$$
\begin{array}{lrr}
q & \geq & 12 \mathrm{KPa}\left(=250 \mathrm{lb} / \mathrm{ft}^{2}\right) \\
a & \leq & 45 g_{0} \\
\alpha & \geq & 0 \\
\alpha & \leq & 25 \mathrm{deg}
\end{array}
$$

where $g_{0}$ is the gravitational acceleration at sea level. In addition, the following values are used for the coefficients $\left(k_{1}, k_{2}, k_{3}\right)$ and the parameter $\bar{\alpha}$ in the objective functional of Eq. (11):

$$
\begin{aligned}
& k_{1}=1 \\
& k_{2}=18 \\
& k_{3}=20 \\
& \bar{\alpha} \approx 0.207 \mathrm{rad} \approx 11.9 \mathrm{deg}
\end{aligned}
$$

Finally, $N+1=125$ was chosen for this study, i.e. 125 LGL points were used. A summary of the resulting NLP is given as follows:

$$
\begin{aligned}
& \text { \# of Optimization Variables }=1251 \\
& \# \text { of Nonlinear Equality Constraints }=1000 \\
& \# \text { of Linear Equality Constraints }=12 \\
& \# \text { of Nonlinear Inequality Constraints }=250
\end{aligned}
$$

The optimization variables are split as follows: 1000 variables correspond to the components of the augmented state at the LGL points, 250 variables correspond to the components of the augmented control at the LGL points, and 1 variable corresponds to the terminal time. Furthermore, the 1000 nonlinear equality constraints correspond to the eight discretized differential equations at the LGL points while the 12 linear equality constraints correspond to the boundary conditions. Finally, the 250 nonlinear inequality constraints correspond to the dynamic pressure and sensed acceleration at the LGL points.

\section{Numerical Optimization via SNOPT}

While in principle any nonlinear numerical optimization algorithm can be used to solve the NLP that arises from the Legendre pseudospectral method, it is preferable to use a method that is computationally efficient. For the current problem (and for trajectory optimization problems in general) it is known that the nonlinear constraint Jacobian is sparse, i.e. a large percentage of the individual derivatives of the nonlinear constraints with respect the optimization variables are zero. Therefore, it is beneficial to use an optimization algorithm that takes advantage of this sparsity. There are several wellknown numerical optimization methods that solve sparse NLPs including SNOPT ${ }^{4}$ and SPRNLP. ${ }^{5}$ In the current research the numerical optimization was carried out with $\mathrm{SNOPT}^{4}$ version 6.1 in FORTRAN. The objective function gradient and nonlinear constraint Jacobian were computed analytically. Furthermore, default feasibility and optimality tolerances were used. (see the SNOPT User's Manual for further details ${ }^{4}$ ). Finally, the pseudospectral differentiation matrices, pseudospectral weights, and the Legendre-Gauss-Lobatto points that are required in Eqs. (12)-(14) were computed using the FORTRAN software PseudoPack. ${ }^{12}$

\section{$\underline{\text { Results }}$}

The results obtained by solving the NLP of Eq. (12)Eq. (14) are shown in Fig. 1-Fig. 7. In addition, the solution obtained via numerical integration of the differential equations using the controls $u_{\alpha}$ and $u_{\sigma}$ obtained from the Legendre pseudospectral method is plotted alongside the NLP solution. The numerical integration was performed in MATLAB using the lsode integrator ${ }^{13}$ in FORTRAN with a relative error tolerance of $10^{-12}$.

The results illustrate several key features of the optimal trajectory. First, it is seen from Fig. 1 that the altitude does not decrease monotonically along the optimal trajectory, but actually increases twice (thus attaining two local maximum) during flight. The first rise in altitude (which begins at the initial state) enables the vehicle to fly in a low density region (because the altitude is greater than $40 \mathrm{~km}$ ) in order 
to achieve the required range (2800 $\mathrm{km}$ in this case). During the subsequent fall in altitude, the speed of the vehicle is reduced so that the vehicle can arrive at the target with the prescribed terminal speed of 1219 $\mathrm{m} / \mathrm{s}$. Furthermore, it is seen from Fig. 2 that the two local maxima in altitude occur at points where the dynamic pressure constraint is active (i.e. when $q=12 \mathrm{kPa}$ ). Therefore, these local maxima in altitude are constrained by the lower limit on dynamic pressure. In particular, if there was no constraint on dynamic pressure, the vehicle would attain a higher altitude at each of these maxima.

The second key feature of the results is exhibited by the Earth relative downtrack and Earth relative crosstrack as shown in Fig. 3 (see Appendix for the definition of downtrack and crosstrack). It is seen from Fig. (3) that the vehicle steers approximately $500 \mathrm{~km}$ in the crosstrack direction and arrives at the target nearly orthogonal to the reference downtrack plane (see Appendix). More specifically, from Fig. 3 it is seen that the vehicle arrives at the target from slightly behind.

The third key feature of the results is the behavior of the angle of attack which is shown in Fig. 6. It can be seen that, because of the inclusion of the first term in the cost function of Eq. (11), the angle of attack stays near $\bar{\alpha}=11.9$ deg for a large percentage of the total flight time. However, the angle of attack increases to 23 deg near the end of flight, indicating that a large angle of attack is required in order to attain the target. The reason that the angle of attack increases so dramatically before decreasing to zero is because the vehicle needs to deplete speed at the end of the trajectory in order to arrive at the target with a terminal speed of $1219 \mathrm{~m} / \mathrm{s}$. The only way the vehicle can deplete this speed is by increasing drag which requires a large angle of attack. In fact, it was found that if the first term in the cost functional of Eq. (11) was not present, the vehicle would fly at its maximum angle of attack for a long duration (approximately 30 seconds) as it approached the target. However, as alluded to earlier, in order to maintain two-sided control authority in the event of dispersions in an actual flight, it is undesirable to fly at either the upper or lower angle of attack limit. Therefore, the first term in the cost functional is essential for maintaining control margin.

The last key feature of the results is exhibited by the behavior of the bank angle. It is seen from Fig. 7 that the vehicle arrives at the target with a bank angle of $180 \mathrm{deg}$, i.e. the vehicle is flying upside-down as it arrives at the target. There are two reasons why the bank angle approaches $180 \mathrm{deg}$ as the vehicle arrives at the target. First, the terminal boundary condi- tion on the flight path angle (-89 deg) requires that the lift point downward as the vehicle approaches the target. However, because the vehicle cannot fly with a negative angle of attack, the only way to attain a downward pointing lift direction is via a $180 \mathrm{deg}$ rotation in the bank angle.

\section{Optimality and Accuracy of Solution}

The solution from the Legendre pseudospectral method is now compared to the solution derived from the first-order necessary conditions for optimality. ${ }^{14}$ For the maneuvering re-entry vehicle optimal control problem described above, the Hamiltonian is given as

$$
H=\mathcal{L}+\boldsymbol{\lambda}^{T} f
$$

where $\mathcal{L}$ is Lagrangian of the cost function of Eq. (11), $f$ is the vector field of the differential equations in Eqs. (1) and (6), and $\boldsymbol{\lambda}$ is the co-state. The co-state can be written component-wise as

$$
\boldsymbol{\lambda}=\left(\lambda_{r}, \lambda_{\theta}, \lambda_{\phi}, \lambda_{v}, \lambda_{\gamma}, \lambda_{\psi}, \lambda_{u_{\alpha}}, \lambda_{u_{\sigma}}\right)^{T}
$$

For this problem, extremal controls are found from the conditions

$$
\begin{aligned}
& \partial H / \partial u_{\alpha}=2 k_{2}\left(u_{\alpha}^{*} / u_{\alpha, \max }\right)+\lambda_{u_{\alpha}}=0 \\
& \partial H / \partial u_{\sigma}=2 k_{3}\left(u_{\sigma}^{*} / u_{\sigma, \max }\right)+\lambda_{u_{\sigma}}=0
\end{aligned}
$$

which leads to the following extremal controls:

$$
\begin{aligned}
& u_{\alpha}^{*}=-\frac{u_{\alpha, \max }}{2 k_{2}} \lambda_{u_{\alpha}} \\
& u_{\sigma}^{*}=-\frac{u_{\sigma, \max }}{2 k_{3}} \lambda_{u_{\sigma}}
\end{aligned}
$$

Fig. 8 shows $u_{\alpha}$ vs. $t$ (obtained from the Legendre pseudospectral method) alongside $u_{\alpha}^{*}$ vs. $t$. Similarly, Fig. 9 shows $u_{\sigma}$ vs. $t$ alongside $u_{\sigma}^{*}$ vs. $t$. It can be seen that, for both $u_{\alpha}$ and $u_{\sigma}$, the controls obtained from the Legendre pseudospectral method and those obtained from Eq. (23) are extremely close. Fig. 10 shows the $H$ vs. $t$ (using canonical units in which the results were obtained). Since for this problem the Hamiltonian is not an explicit function of time and the terminal time is free (see Eq. (16), the Hamiltonian along an extremal trajectory (denoted $H^{*}$ ) must be zero. From Fig. 10 it is seen that $H^{*}$ oscillates about zero and its magnitude remains small throughout the trajectory. The results obtained from Figs. 8-10 give strong evidence that the trajectory obtained from the Legendre pseudospectral method is close to an extremal trajectory.

In addition to assessing the proximity to optimality of the Legendre pseudospectral method solution, 
it is important to assess the accuracy of the trajectory obtained via numerical integration with the angle of attack and bank angle obtained from the Legendre pseudospectral method. It can be seen from Fig. 1-Fig. 7 that the solution obtained via numerical integration is extremely close to that obtained via numerical solution of the NLP using the Legendre pseudospectral method, resulting in a trajectory that is virtually indistinguishable from the solution obtained from the Legendre pseudospectral method. More specifically, the differences in downtrack and crosstrack (which are of most importance for this application) at the terminal time between the solution obtained via the Legendre pseudospectral method and the solution obtained via numerical integration are 1.65 meters and 18.08 meters, respectively. These individual errors translate to an overall position error of 18.16 meters at the terminal time. This level of accuracy is quite remarkable considering that the vehicle has traversed a distance of $2800 \mathrm{~km}$ during flight. The results of the numerical integration demonstrate that the pseudospectral solution is of sufficient accuracy that, under perfect flight conditions, the commanded angle of attack and bank angle can be used to steer the vehicle from the initial state to a terminal state that is extremely close to the target.

\section{CONCLUSIONS}

The problem of performance optimization of a maneuvering re-entry vehicle was studied. The objective was to determine an optimal control to steer a high lift-to-drag ratio from an initial state just after atmospheric entry to a terminal state at Earth impact. An objective functional was derived that attempted to maximize the control margin of the vehicle, thus leaving room for unmodeled perturbations during actual flight. The optimal control problem was transcribed to a nonlinear programming problem (NLP) via a Legendre pseudospectral method. The NLP was solved using a sparse nonlinear optimization algorithm. The key features of the optimal trajectory are described and the performance of the pseudospectral method is discussed. Finally, a motivation was given for using the pseudospectral method as a guidance law.

\section{$\underline{\text { ACKNOWLEDGMENTS }}$}

This work was supported under Draper Laboratory Internal Research and Development. The authors would like to acknowledge Professor I. Michael Ross of the Naval Postgraduate School for his helpful suggestions during the early part of this work. The authors would also like to acknowledge Professor Wai Sun Don of Brown University for providing a specialized module for the pseudospectral computations used in this work. Finally, the authors would like to acknowledge Mr. Timothy Brand for providing helpful insight throughout the course of this work.

\section{$\underline{\text { APPENDIX }}$}

\section{EARTH RELATIVE DOWNTRACK \& CROSSTRACK}

The Earth relative downtrack and crosstrack distances are defined as follows. Let $\boldsymbol{r}_{0}$ and $\boldsymbol{r}_{f}$ be the initial and terminal positions of a vehicle during flight expressed in an arbitrary Cartesian Earth-centered Earth-fixed (ECEF) coordinates. Furthermore, let

$$
\begin{gathered}
\boldsymbol{u}_{1}=\boldsymbol{r}_{0} /\left\|\boldsymbol{r}_{0}\right\|_{2} \\
\boldsymbol{u}_{3}=\left(\boldsymbol{u}_{1} \times \boldsymbol{r}_{f}\right) /\left\|\boldsymbol{u}_{1} \times \boldsymbol{r}_{f}\right\|_{2}
\end{gathered}
$$

and

$$
\boldsymbol{u}_{2}=\boldsymbol{u}_{3} \times \boldsymbol{u}_{1}
$$

Then the transformation from the downtrackcrosstrack coordinate system to the ECEF coordinate system is given as

$$
\boldsymbol{T}_{d c 2 e}=\left[\begin{array}{lll}
\boldsymbol{u}_{1} & \boldsymbol{u}_{2} & \boldsymbol{u}_{3}
\end{array}\right]
$$

where the $\boldsymbol{u}_{1}-\boldsymbol{u}_{2}$ plane is the Earth relative downtrack plane while the $\boldsymbol{u}_{1}-\boldsymbol{u}_{3}$ plane is the Earth relative crosstrack plane relative to the coordinate system $\left(\boldsymbol{u}_{1}, \boldsymbol{u}_{2}, \boldsymbol{u}_{3}\right)$. Using Eq. (27), the transformation from the original ECEF coordinate system to the downtrack-crosstrack coordinate system is given as $\boldsymbol{T}_{e 2 d c}=\boldsymbol{T}_{d c 2 e}^{T}\left(\right.$ where $(\cdot)^{T}$ denotes matrix transpose). Using the transformation $\boldsymbol{T}_{e 2 d c}$, the downtrack distance, $d$, and the crosstrack distance, $c$, are computed as follows. Let $\boldsymbol{r}$ be the position of the vehicle in original ECEF coordinates at any point along 
a trajectory. Then the position in the coordinate system $\left(\boldsymbol{u}_{1}, \boldsymbol{u}_{2}, \boldsymbol{u}_{3}\right)$, denoted $\boldsymbol{r}_{d c}$, is given as

$$
\boldsymbol{r}_{d c}=\boldsymbol{T}_{e 2 d c} \boldsymbol{r}
$$

The vector $\boldsymbol{r}_{d c}$ can be expressed in spherical coordinates as

$$
\boldsymbol{r}_{d c}=\left[\begin{array}{c}
\cos b \cos a \\
\cos b \sin a \\
\sin b
\end{array}\right]
$$

where $a$ is the Earth relative downrange angle (in radians) and $b$ is the Earth relative crossrange angle (in radians) as measured in the coordinate system $\left(\boldsymbol{u}_{1}, \boldsymbol{u}_{2}, \boldsymbol{u}_{3}\right)$. The Earth relative downtrack distance (or Earth relative downrange distance) and Earth relative crosstrack distance (or Earth relative crossrange distance) are then given as

$$
\begin{aligned}
& d=R_{e} a \\
& c=R_{e} b
\end{aligned}
$$

where $R_{e}$ is the equatorial radius of the Earth and is taken as the reference length.

\section{References}

${ }^{1}$ Regan, F. J., and Anandakrishnan, S. M., Dynamics of Atmospheric Re-Entry, American Institute of Aeronautics and Astronautics, Washington, D.C., 1993.

${ }^{2}$ Vinh, N. X., Busemann, A., and Culp, R. D., Hypersonic and Planetary Entry Flight Mechanics, University of Michigan Press, Ann Arbor, MI, 1980.

${ }^{3}$ Slivinsky, S., Galloway, R., Breitling, S., and Wilborn, C., "Missile Technology Demonstration 3 (MTD-3) Demonstrating Global Position System (GPS) Technology in an Application to Range Safely Tracking and Precision Guidance, Navigation, and Control, Proceedings of the AIAA Space Technology Conference and Exposition, AIAA-994432, Albuquerque, NM, 28-30 September 1999.

${ }^{4}$ Gill, P. E., Murray, W., Saunders, M. A., User's Guide for SNOPT 5.3: A FORTRAN Package for Large-Scale Nonlinear Programming, 1998.
${ }^{5}$ Betts, J. T. and Huffman, W. P., "Sparse Optimal Control Software - SOCS," Mathematics and Engineering Analysis Library Report, MEA-LR-085, Boeing Information and Support Services, P. O. Box 3797, Seattle, WA, 98124-2297, 15 July 1997.

${ }^{6}$ Fahroo, F. and Ross, I. M., "Costate Estimation by a Legendre Pseudospectral Method", Journal of Guidance, Control, and Dynamics, Vol. 24, No. 2, March-April 2001, pp. 270-277.

${ }^{7}$ Fahroo, F., and Ross, I. M., "A Spectral Patching Method for Direct Trajectory Optimization," The Journal of the Astronautical Sciences, Vol. 48, No. 2 and 3, April-September, 2000.

${ }^{8}$ Fahroo, F., and Ross, I. M., "Direct Trajectory Optimization by a Chebyshev Pseudospectral Method," Journal of Guidance, Control, and Dynamics, Vol. 25, No. 1, January-February 2001, pp. $160-165$.

${ }^{9}$ Betts, J. T., "Survey of Numerical Methods for Trajectory Optimization," Journal of Guidance, Control, and Dynamics, Vol. 21, No. 2, March-April 1998, pp. 193-207.

${ }^{10}$ Vlases, W. G., Paris, S. W., Lajoie, R. M., Martens, M. J., and Hargraves, C. R., "Optimal Trajectories by Implicit Simulation," Boeing Aerospace and Electronics, Technical Report WRDC-TR-90-3056, Wright-Patterson Air Force Base, 1990.

${ }^{11}$ Bate, R. R., Mueller, D. D., and White, J. E., Fundamental of Astrodynamics, Dover Publications, New York, 1971.

${ }^{12}$ Don, W. S., and Costa, B., Scientific Computing with Pseudospectral Methods and the PseudoPack Library, 2000.

${ }^{13}$ Hindmarsh, A. C., ODEPACK: A Systematized Collection of ODE Solvers in Scientific Computing, R. S. Stepleman, et. al. (eds.), North-Holland, Amsterdam, 1983, pp. 55-64.

${ }^{14}$ Athans, M., and Falb, P. L., Optimal Control, McGraw-Hill, New York, 1966. 


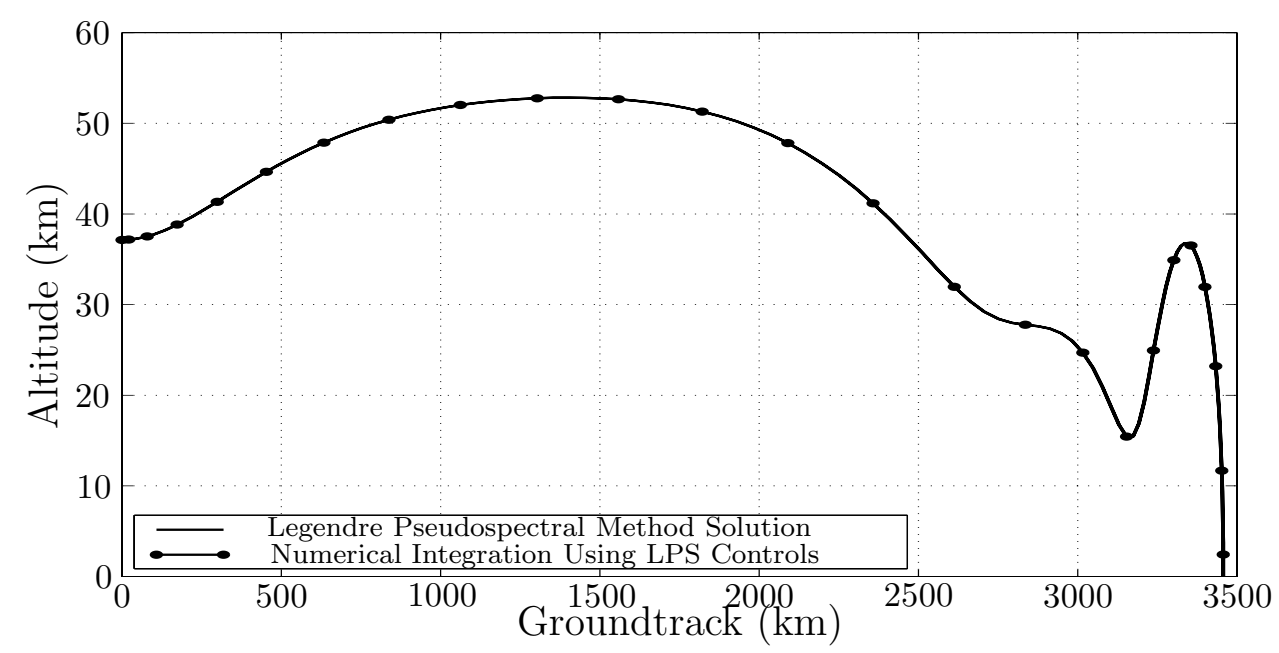

Figure 1: Altitude vs. groundtrack from LPS method of Ref. 6 alongside numerically integrated solution.

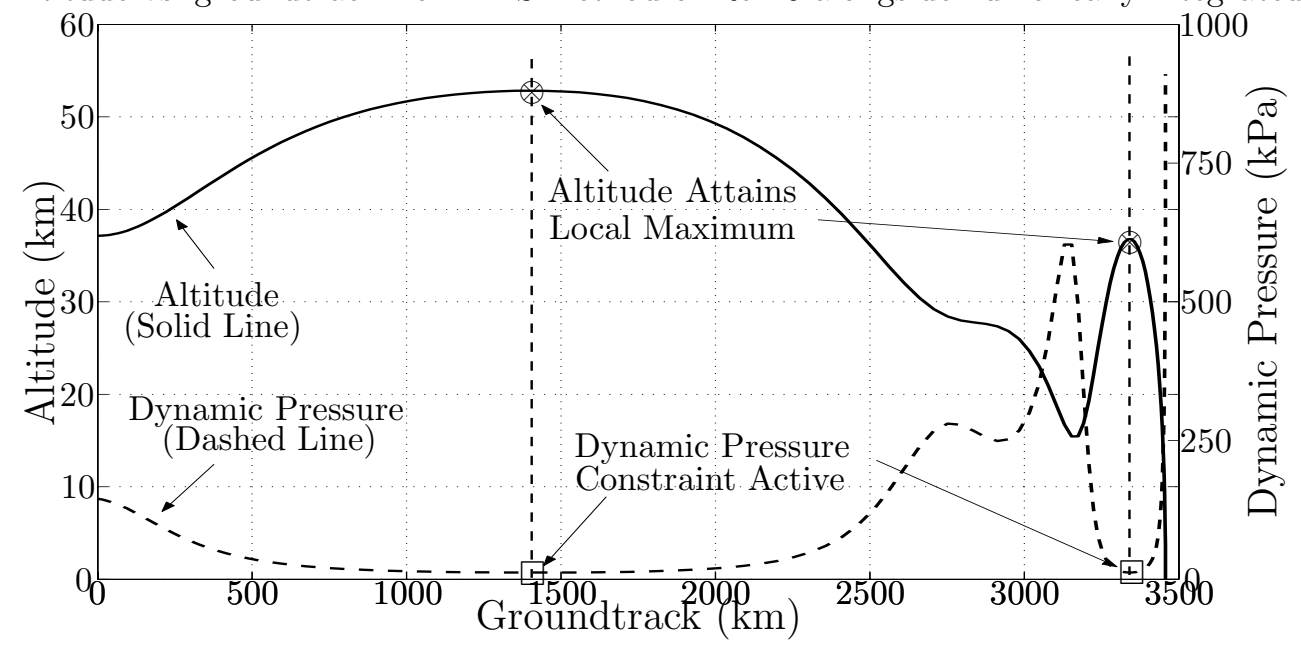

Figure 2: Altitude and dynamic pressure vs. groundtrack from LPS method of Ref. 6 .

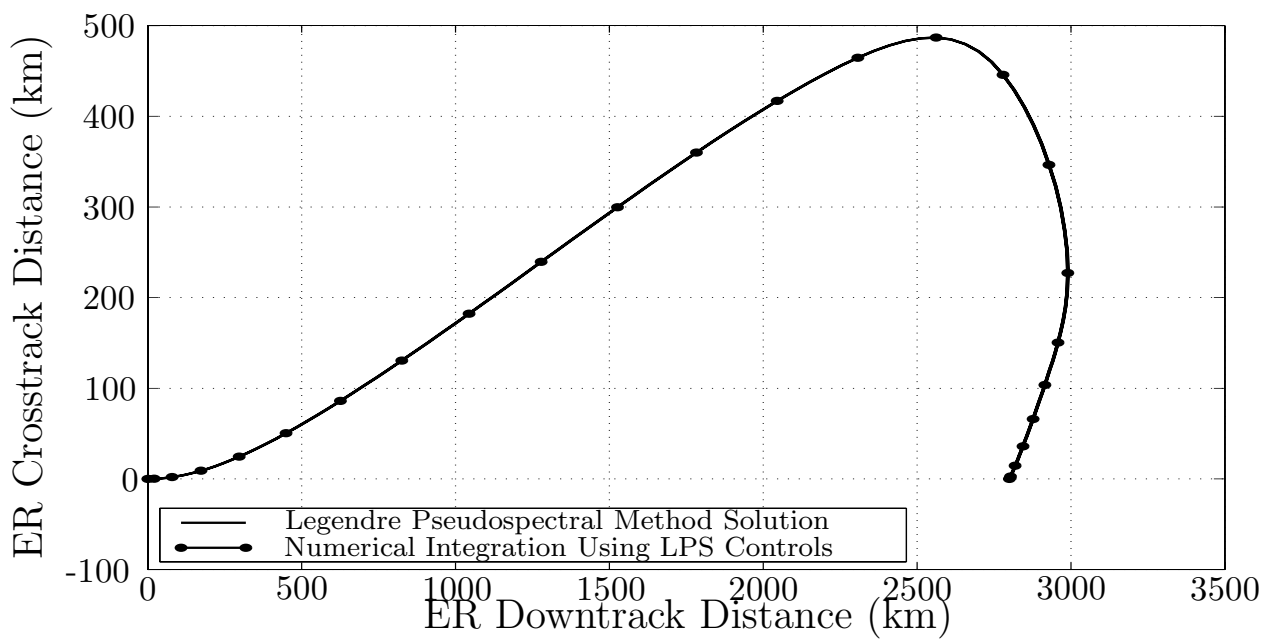

Figure 3: Earth relative (ER) Downtrack distance vs. Earth relative crosstrack distance from LPS method of Ref. 6 alongside numerically integrated solution. 


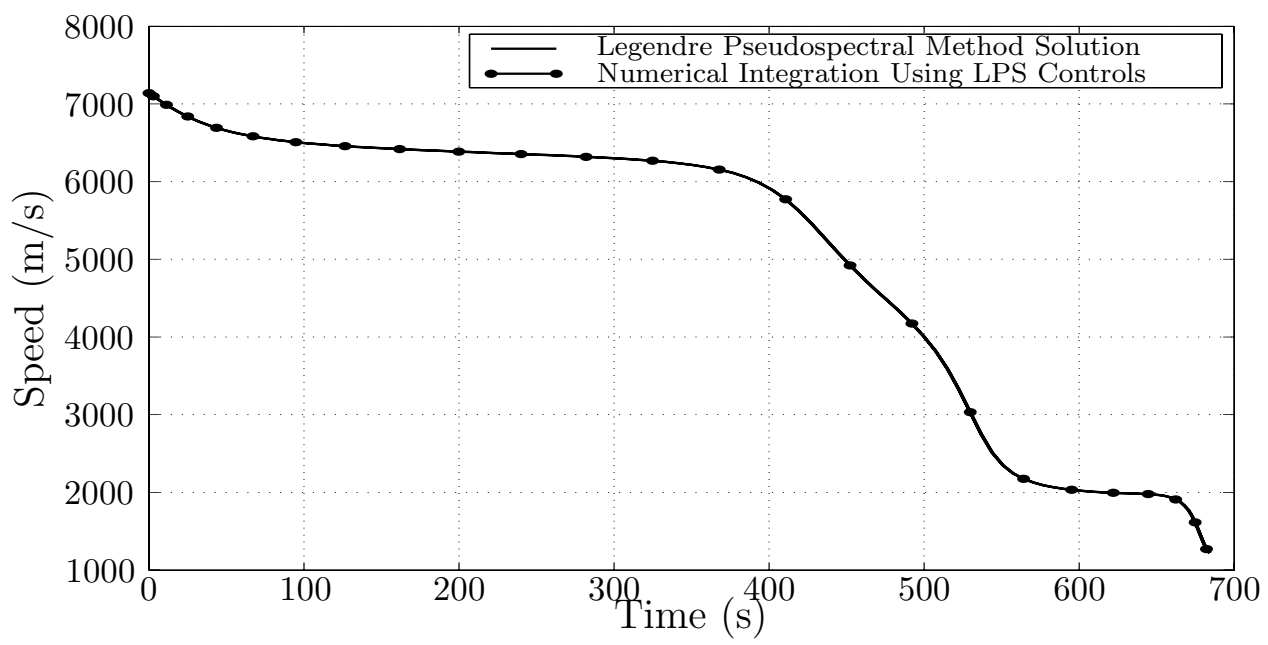

Figure 4: Speed vs. time from LPS method of Ref. 6 alongside numerically integrated solution.

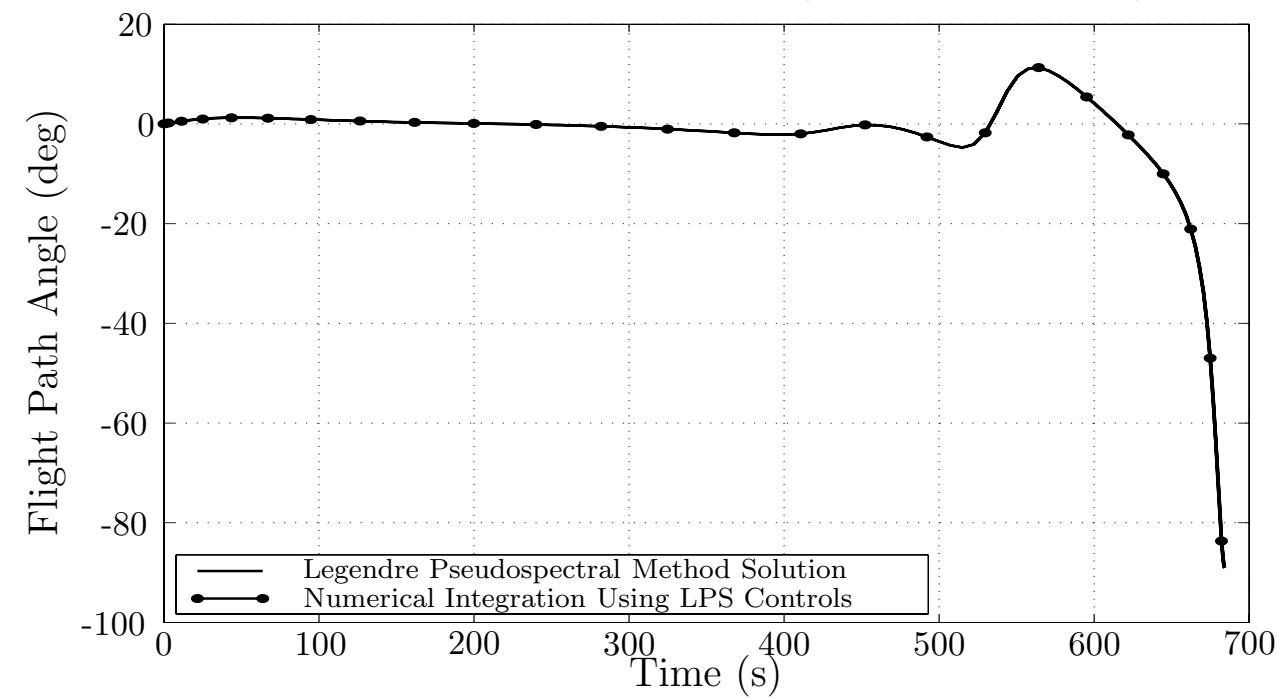

Figure 5: Flight path angle vs. time from LPS method of Ref. 6 alongside numerically integrated solution.

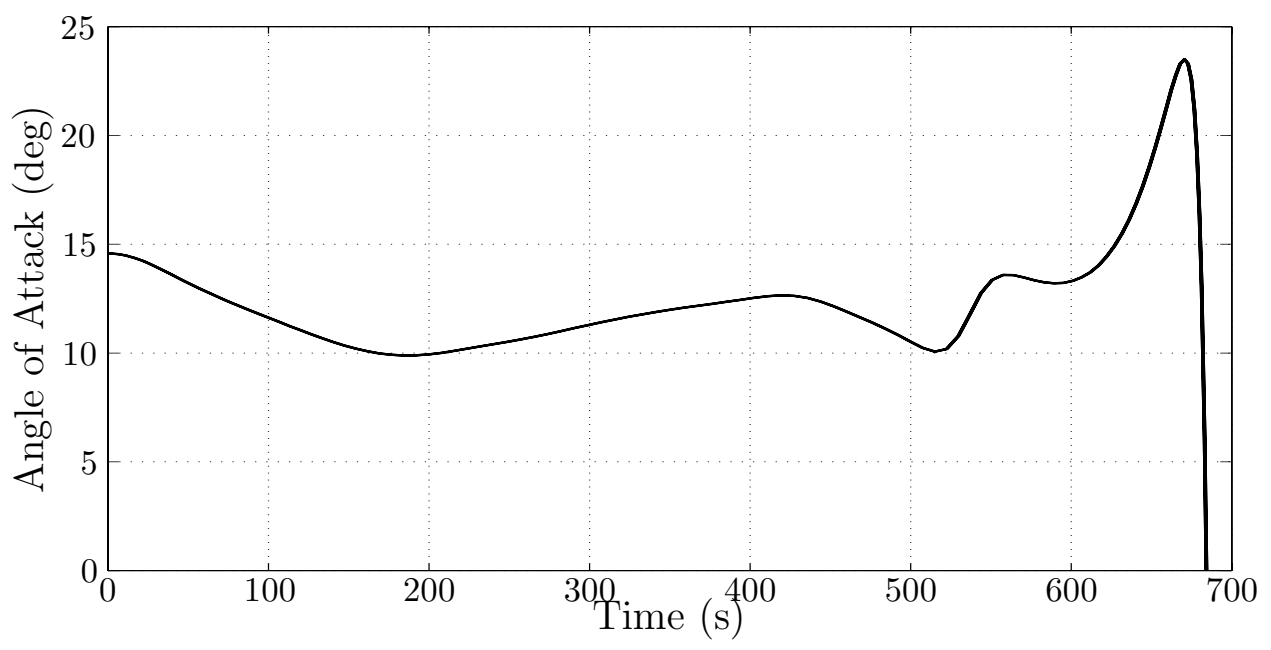

Figure 6: Angle of attack vs. time from LPS method of Ref. 6. 


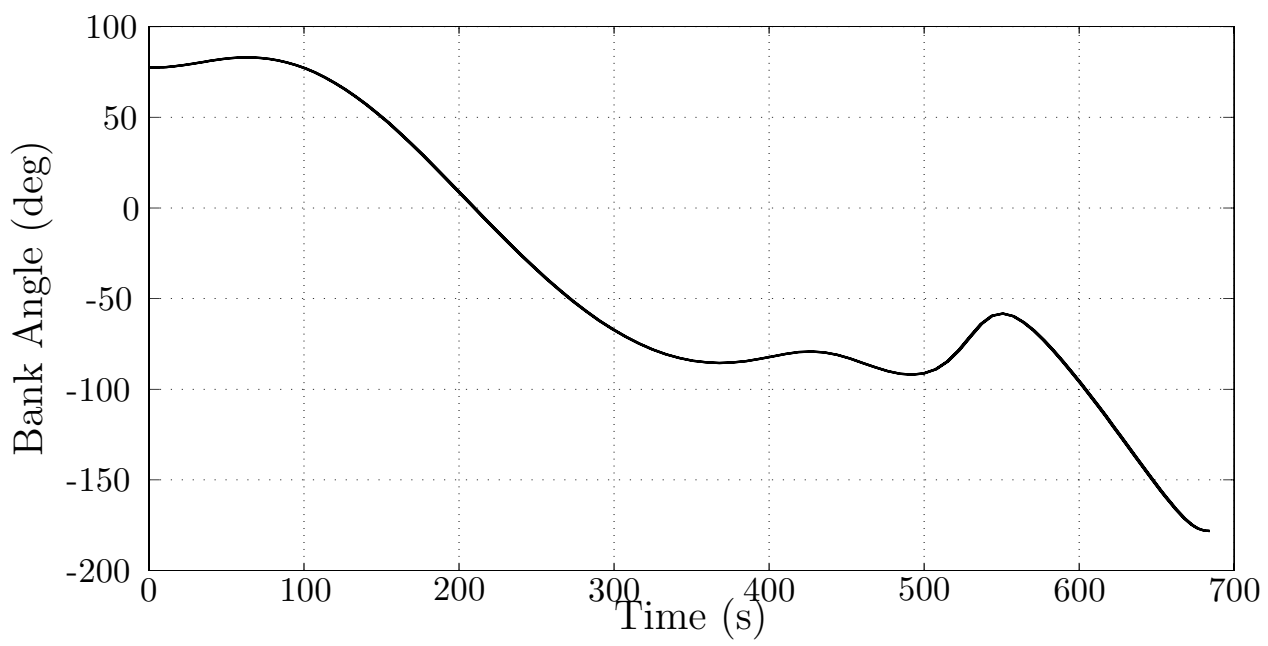

Figure 7: Bank angle vs. time from LPS method of Ref. 6 .

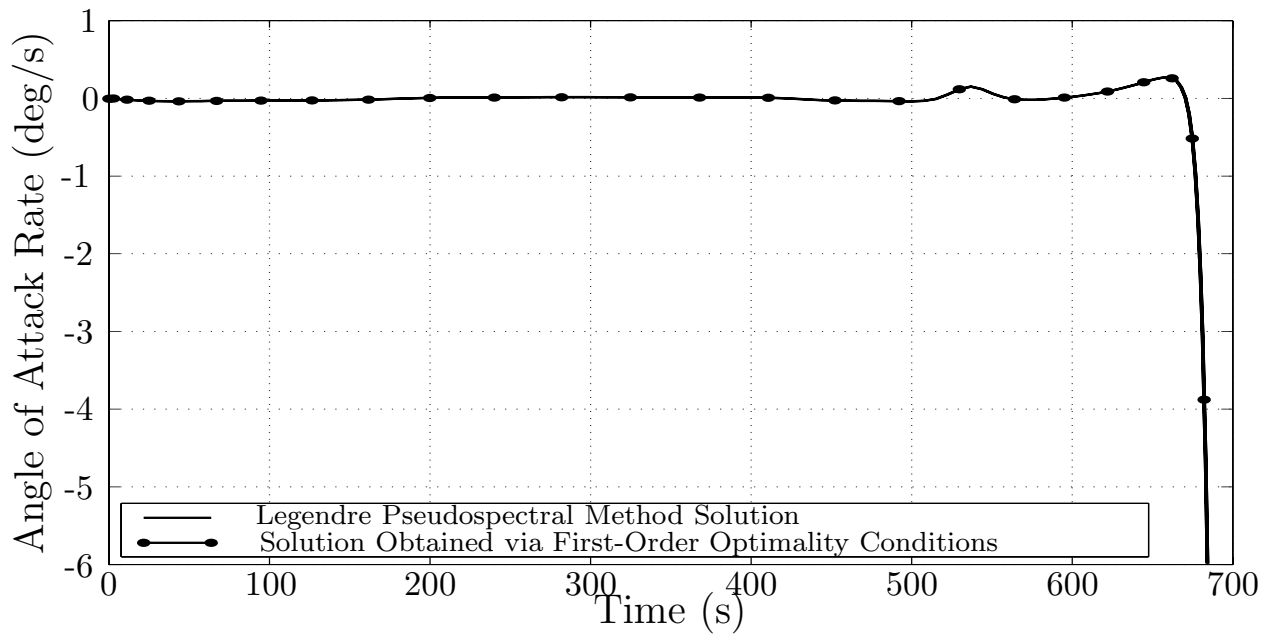

Figure 8: Angle of attack rate vs. time from LPS method of Ref. 6 alongside solution found from first-order optimality conditions.

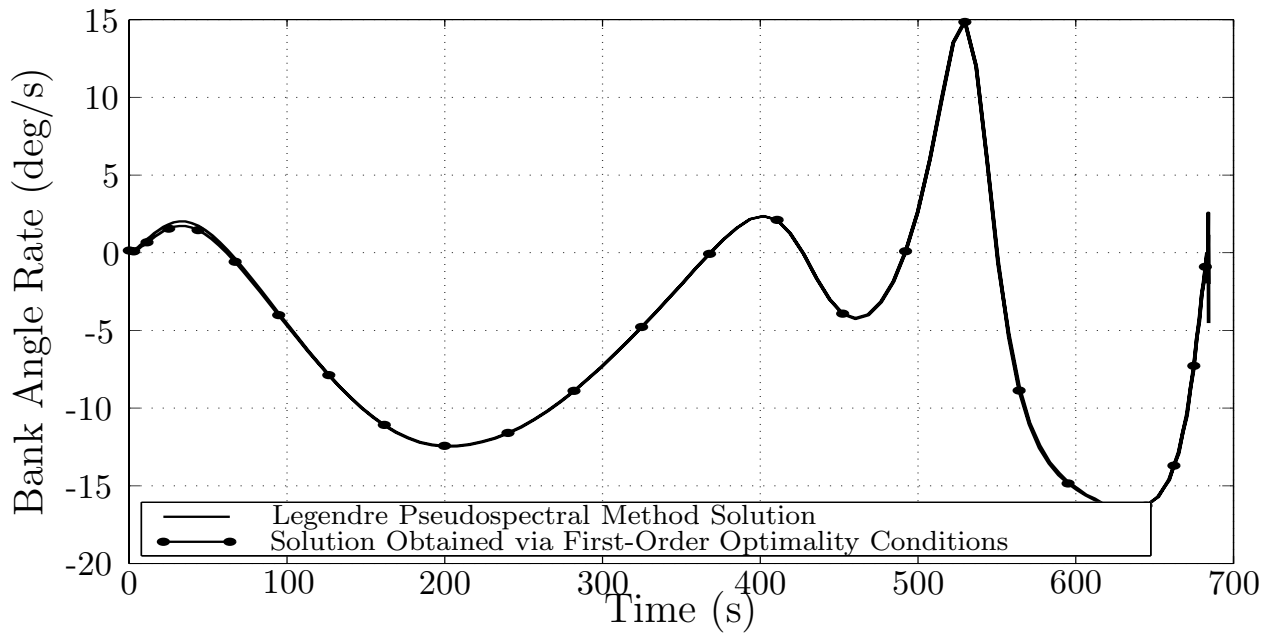

Figure 9: Bank angle rate vs. time from LPS method of Ref. 6 alongside solution found from first-order optimality conditions. 


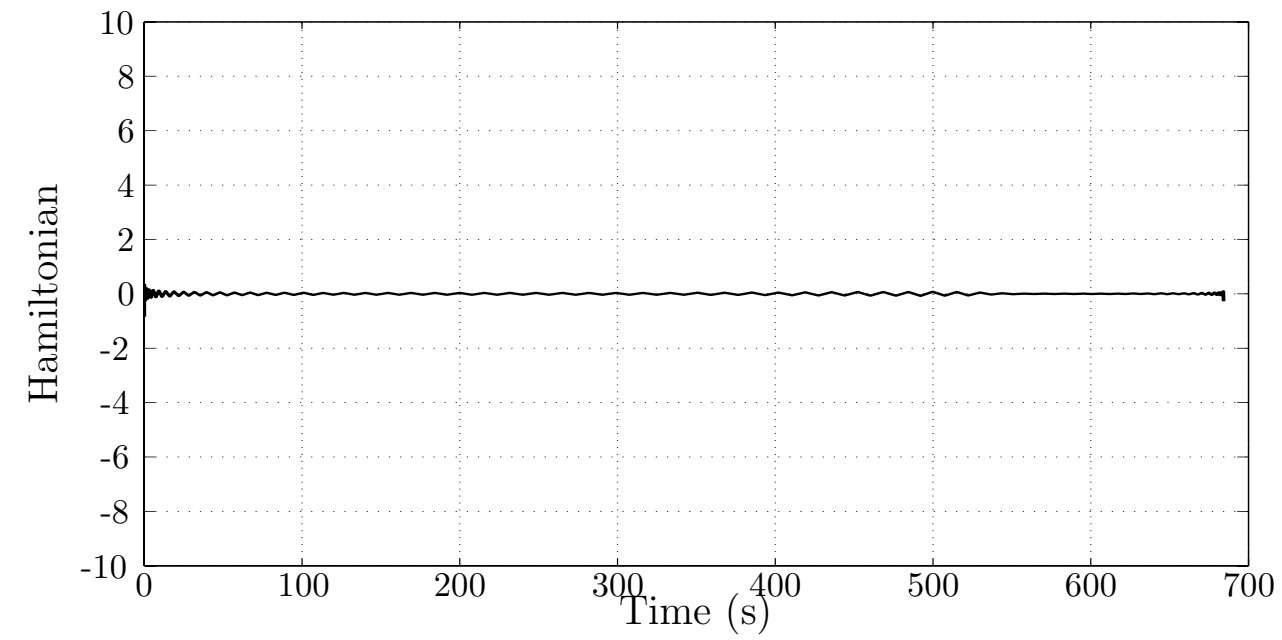

Figure 10: Hamiltonian vs. time computed using the co-state estimates obtained from the LPS method of Ref. 6. 